\title{
Modeling the trade-off between transmissibility and contact in infectious disease dynamics
}

\author{
Chiu-Ju Lin ${ }^{\mathrm{a}}$, Kristen A. Deger ${ }^{\mathrm{a}}$, Joseph H. Tien ${ }^{\mathrm{a}}$ \\ ${ }^{a}$ Department of Mathematics, The Ohio State University, Columbus, OH 43210, USA
}

\begin{abstract}
Symptom severity affects disease transmission both by impacting contact rates, as well as by influencing the probability of transmission given contact. This involves a trade-off between these two factors, as increased symptom severity will tend to decrease contact rates, but increase the probability of transmission given contact (as pathogen shedding rates increase with symptom severity). This paper explores this trade-off between contact and transmission given contact, using a simple compartmental susceptibleinfected-recovered type model. Under mild assumptions on how contact and transmission probability vary with symptom severity, we give sufficient, biologically intuitive criteria for when the basic reproduction number varies nonmonotonically with symptom severity. Multiple critical points are possible. We give a complete characterization of the region in parameter space where multiple critical points are located in the special case where contact rate decreases exponentially with symptom severity. We consider a multi-strain version of the model with complete cross-immunity and no super-infection. In this model, we prove that the strain with highest basic reproduction number drives the other strains to extinction. This has both evolutionary and epidemiological implications, including the possibility of an intervention paradoxically resulting in increased infection prevalence.
\end{abstract}

Keywords: competitive exclusion principle, adaptive dynamics, symptom severity, evolution of virulence, evolutionary bistability

\section{Introduction}

Many pathogens exhibit a wide range of symptom severity following infection, ranging from asymptomatic to severe. How does this variation affect 
disease dynamics? Symptom severity is often correlated with pathogen loads and shedding rates $[2,13,15,19,22,25,30,31]$ and thus to the probability of disease transmission given contact with a susceptible individual. On the other hand, disease symptoms will also influence contact rates of infected individuals, for example due to illness affecting an individual's ability to attend school, go to work, travel, or have sexual encounters. There is thus a trade-off between transmissibility and contact, similar to the classic trade-off between transmission and host mortality in the study of pathogen virulence evolution $[2,6,7,14,15,20]$. Understanding this trade-off has both evolutionary and epidemiological implications, for example regarding strain competition and pathogen virulence, and for evaluating control strategies such as vaccination and chemotherapy.

The purpose of this paper is to examine these questions using a simple mathematical model, with the following basic assumptions: (i) infection can result in a range of symptoms, from mild to severe; (ii) the probability of transmission given contact is a monotone increasing function of symptom severity; (iii) contact rate is a monotone decreasing function with respect to symptom severity. To biologically motivate these assumptions, note that variation in symptoms is observed across taxa and disease transmission routes. Specific examples include El Tor cholera, with $75 \%$ asymptomatic, $23 \%$ mild or moderate, and $2 \%$ severe infection [19]; pertussis, where age and immunization status influence symptom severity [21]; influenza, where neuraminidase inhibitors can reduce symptom severity and duration [23]; herpes simplex virus (HSV), with frequent subclinical viral shedding [31], and many more. For (ii), pathogen shedding rates are often correlated with symptoms, both in magnitude (e.g. $10^{3}$ vibrios per gram of stool for asymptomatic cholera patients [25], versus $10^{7}-10^{8}$ vibrios per gram of stool for severely symptomatic individuals [19]) and frequency (e.g. symptomatic individuals with HSV experienced more frequent shedding than asymptomatic individuals [31]). Shedding rates relate to transmission probabilities through dose-response curves, which are typically monotone functions of dose [18]. Specifics on how variation in shedding rates vary with symptom severity, and how this translates into corresponding variation in transmission probability, will depend upon the shape of the dose-response curve for the pathogen in question. For example, asymptomatic transmission of norovirus is believed widespread, due to comparable shedding rates of asymptomatic and symptomatic individuals, together with a very low infectious dose [26]. Regarding (iii), certain types of contact clearly decrease with symptom severity, for 
example illness-induced absences from school or the workplace. Recent empirical studies examining how illness affects contact patterns include Chen et al [9] and van Kerckhove et al [34].

The model we consider is a simple extension of the classical susceptibleinfected-recovered (SIR) ordinary differential equation model. The infected compartment is divided into two compartments, corresponding to infected individuals with mild $\left(I_{m}\right)$ or severe $\left(I_{s}\right)$ disease symptoms. This model has been considered by others, for example by Brauer et al [5] and Vivas-Barber et al [36] in the context of influenza dynamics. What is new here is our consideration of the trade-off between contact and transmissibility in this setting. Specifically, we use this model to examine under what conditions this trade-off selects for intermediate levels of pathogen virulence, and what are the corresponding evolutionary and epidemiological implications. In particular, we find conditions for which $\mathcal{R}_{0}$ exhibits local maxima at intermediate symptom severity, and prove a competitive exclusion principle showing that for this model the pathogen strain with highest basic reproduction number will drive the other strains to extinction.

The remainder of this paper is organized as follows. Section 2 presents the basic model together with analysis of the equations, including computation of $\mathcal{R}_{0}$ and proving global stability for the system. In section 3 , we explore the effects of the trade-off between contact and transmissibility on $\mathcal{R}_{0}$ in detail. In particular, we give conditions for when $\mathcal{R}_{0}$ varies non-monotonically with symptom severity, and discuss the number of critical points that can arise. Section 4 extends the model to include multiple pathogen strains. We prove that the highest $\mathcal{R}_{0}$ strain will drive the other strains to extinction. We also present an adaptive dynamics treatment of virulence evolution, together with simulations of evolutionary dynamics. The paper concludes with a discussion in section 5 .

\section{Model}

We consider a simple extension of the basic susceptible-infected-recovered (SIR) framework by differentiating between mild $\left(I_{m}\right)$ and severe $\left(I_{s}\right)$ infection. A fraction $f$ of infected individuals experience mild symptoms and enter the $I_{m}$ compartment following infection, with the remaining fraction $1-f$ entering the $I_{s}$ class. Individuals experiencing mild versus severe symptoms have potentially different infectious periods $\left(1 / \gamma_{m}\right.$ and $1 / \gamma_{s}$, respectively), and transmission parameters $\beta_{m}, \beta_{s}$. A flow diagram of the model is given 
in Figure 1. This model has been introduced previously in the literature, for example by Vivas-Barber et al [36]. What is novel here is consideration of how symptom severity affects the transmission parameters $\beta_{m}$, $\beta_{s}$, through the trade-off between contact and transmission given contact. The dynamics

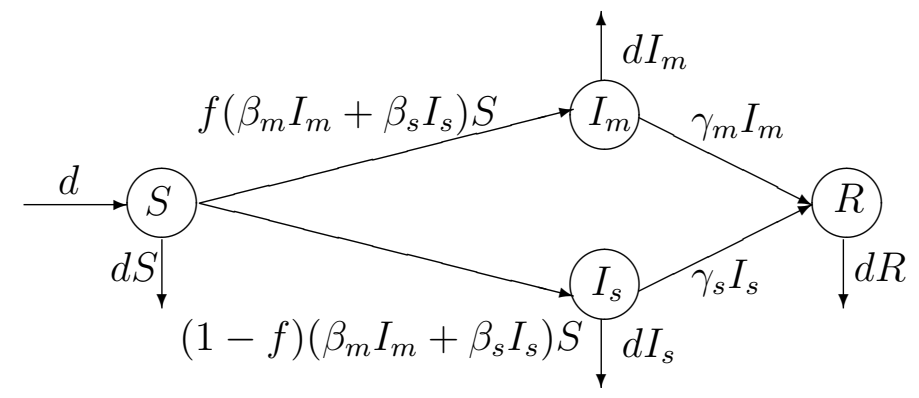

Figure 1: Flow diagram of the $S I_{m} I_{s} R$ model (1).

comply with the following equations:

$$
\begin{aligned}
\dot{S} & =d-d S-S\left(\beta_{m} I_{m}+\beta_{s} I_{s}\right), \\
\dot{I}_{m} & =f S\left(\beta_{m} I_{m}+\beta_{s} I_{s}\right)-\left(\gamma_{m}+d\right) I_{m}, \\
\dot{I}_{s} & =(1-f) S\left(\beta_{m} I_{m}+\beta_{s} I_{s}\right)-\left(\gamma_{s}+d\right) I_{s},
\end{aligned}
$$

where ${ }^{\cdot}=\frac{d}{d t}$. Here we assume the population has a constant birth rate and natural death rate $d$. Because the equations of $S, I_{m}, I_{s}$ are independent of the variable $R$, we only focus on them and ignore the dynamics of $R$. We further assume the total population size $N$ is constant, let $N=S+I_{m}+$ $I_{s}+R=1$ after scaling. Thus all variables are fractions of the population. We consider the initial condition $\left(S, I_{m}, I_{s}\right)(0) \in \Omega$, where

$$
\Omega:=\left\{\left(S, I_{m}, I_{s}\right) \in[0,1]^{3}: S>0, S+I_{m}+I_{s} \leq 1\right\} .
$$

The solutions of (1) with initial condition in $\Omega$ are positive and bounded, i.e., the system is well defined.

To understand how the contact-transmission trade-off affects the parameters $\beta_{m}, \beta_{s}$ in (1), we will use the probability $p$ of transmission given contact as a surrogate for symptom severity, allowing expression of $\beta$ as a function of $p$. Then the force of infection term is $c(p) p I$, where the contact rate $c(p)$ depends upon the probability of transmission. The transmission parameters $\beta$ can thus be written as the product of two factors, the contact rate $c$, times the probability $p$ of transmission given contact with a susceptible individual. 
To incorporate the trade-off between contact and transmission given contact, we assume that $p$ is a monotone increasing function of symptom severity, and $c$ is monotone decreasing with symptom severity. Let $p$ denote the transmission probability for individuals in $I_{s}$, and let $\sigma p$ denote the transmission probability for individuals in $I_{m}$, where $0 \leq \sigma \leq 1$. The resulting transmission parameters for the mild and severe symptom classes are then:

$$
\begin{aligned}
\beta_{m} & =c(\sigma p) \sigma p \\
\beta_{s} & =c(p) p .
\end{aligned}
$$

The trade-off between transmissibility and contact affects the transmission parameters $\beta_{m}, \beta_{s}$ (cf. (2)). To understand the corresponding effect on the dynamics of system (1), we will focus on how the trade-off affects the basic reproduction number $\mathcal{R}_{0}$. In fact we will see that $\mathcal{R}_{0}$ determines both the long term dynamics of system (1) (Theorem 1), as well as the outcome of multi-strain competition (Theorem 2). We first establish some preliminary facts about system $(1)$. Let $E_{0}=(1,0,0)$ denote the disease-free equilibrium (DFE). Using the next generation matrix approach to compute $\mathcal{R}_{0}[11,33]$ gives:

$$
\mathcal{R}_{0}=f \frac{\beta_{m}}{\gamma_{m}+d}+(1-f) \frac{\beta_{s}}{\gamma_{s}+d}=f \mathcal{R}_{01}+(1-f) \mathcal{R}_{00}
$$

where $\mathcal{R}_{01}$ and $\mathcal{R}_{00}$ are the basic reproduction numbers corresponding to $f=$ 1 and $f=0$, respectively. Hence $\mathcal{R}_{0}$ is a linear combination of $\mathcal{R}_{01}$ and $\mathcal{R}_{00}$. When $\mathcal{R}_{0}>1$, there exists an endemic equilibrium (EE) $E^{*}=\left(S^{*}, I_{m}^{*}, I_{s}^{*}\right)$ with

$$
S^{*}=\frac{1}{\mathcal{R}_{0}}, I_{m}^{*}=\frac{f}{\gamma_{m}+d} d\left(1-\frac{1}{\mathcal{R}_{0}}\right), I_{s}^{*}=\frac{1-f}{\gamma_{s}+d} d\left(1-\frac{1}{\mathcal{R}_{0}}\right) .
$$

From the following theorem we know that the global behavior of (1) is determined by the basic reproduction number $\mathcal{R}_{0}$.

Theorem 1. Suppose $d>0, \beta_{m}, \beta_{s} \geq 0, \gamma_{m}, \gamma_{s}>0$ and $f \in[0,1]$. The dynamics of (1) with initial condition in $\Omega$ is one of the following scenarios:

(a) If $\mathcal{R}_{0} \leq 1$, then the disease free equilibrium $E_{0}$ is globally stable.

(b) If $\mathcal{R}_{0}>1$, then the endemic equilibrium $E^{*}$ is globally stable. 
Proof. We first study the case that $f \in(0,1)$. For (a), consider the Lyapunov function

$$
L(t)=\mu I_{m}+\nu I_{s},
$$

where $\mu, \nu>0$ are constants to be determined later. Thus

$$
\dot{L}(t)=\left(\beta_{m} I_{m}+\beta_{s} I_{s}\right)[\mu f+\nu(1-f)] S-\left[\mu\left(\gamma_{m}+d\right) I_{m}+\nu\left(\gamma_{s}+d\right) I_{s}\right] .
$$

Setting $\mu=\frac{\beta_{m}}{\gamma_{m}+d}$ and $\nu=\frac{\beta_{s}}{\gamma_{s}+d}$ gives

$$
\begin{aligned}
\dot{L} & =\left(\beta_{m} I_{m}+\beta_{s} I_{s}\right)\left(\left[\frac{\beta_{m}}{\gamma_{m}+d} f+\frac{\beta_{s}}{\gamma_{s}+d}(1-f)\right] S-1\right) \\
& \leq\left(\beta_{m} I_{m}+\beta_{s} I_{s}\right)\left(\mathcal{R}_{0}-1\right) \leq 0 .
\end{aligned}
$$

In the set

$$
M=\left\{\left(S, I_{m}, I_{s}\right): \dot{L}=0\right\}=\{(S, 0,0)\},
$$

the maximal invariant set of $M$ is $\left\{E_{0}\right\}$. Hence, by LaSalle's invariance principle, $E_{0}$ is globally stable when $\mathcal{R}_{0} \leq 1$.

To prove (b), note that $\mathcal{R}_{0}>1$ implies the existence of $E^{*}$ and, by [33], instability of $E_{0}$. Consider the Lyapunov function

$$
L(t)=\int_{S^{*}}^{S} \frac{\eta-S^{*}}{\eta} d \eta+\mu \int_{I_{m}^{*}}^{I_{m}} \frac{\eta-I_{m}^{*}}{\eta} d \eta+\nu \int_{I_{s}^{*}}^{I_{s}} \frac{\eta-I_{s}^{*}}{\eta} d \eta,
$$

where $\mu, \nu>0$ are constants to be determined later. From the equations of (1) at equilibrium state $E^{*}$, we have that

$$
\begin{aligned}
\dot{L}= & \left(S-S^{*}\right)\left[d\left(\frac{1}{S}-\frac{1}{S^{*}}\right)-\left(\beta_{m} I_{m}+\beta_{s} I_{s}\right)+\left(\beta_{m} I_{m}^{*}+\beta_{s} I_{s}^{*}\right)\right] \\
& +\mu\left(I_{m}-I_{m}^{*}\right)\left[f S\left(\beta_{m}+\beta_{s} \frac{I_{s}}{I_{m}}\right)-f S^{*}\left(\beta_{m}+\beta_{s} \frac{I_{s}^{*}}{I_{m}^{*}}\right)\right] \\
& +\nu\left(I_{s}-I_{s}^{*}\right)\left[(1-f) S\left(\beta_{m} \frac{I_{m}}{I_{s}}+\beta_{s}\right)-(1-f) S^{*}\left(\beta_{m} \frac{I_{m}^{*}}{I_{s}^{*}}+\beta_{s}\right)\right] \\
= & -\frac{d}{S S^{*}}\left(S-S^{*}\right)^{2}+\beta_{m}\left(S-S^{*}\right)\left(I_{m}^{*}-I_{m}\right)+\beta_{s}\left(S-S^{*}\right)\left(I_{s}^{*}-I_{s}\right) \\
& +\mu f \beta_{m}\left(I_{m}-I_{m}^{*}\right)\left(S-S^{*}\right)+\mu f \beta_{s}\left(I_{m}-I_{m}^{*}\right)\left[\frac{S I_{s}}{I_{m}}-\frac{S^{*} I_{s}^{*}}{I_{m}^{*}}\right] \\
& +\nu(1-f) \beta_{s}\left(I_{s}-I_{s}^{*}\right)\left(S-S^{*}\right)+\nu(1-f) \beta_{m}\left(I_{s}-I_{s}^{*}\right)\left[\frac{S I_{m}}{I_{s}}-\frac{S^{*} I_{m}^{*}}{I_{s}^{*}}\right] .
\end{aligned}
$$


Note that

$$
\begin{aligned}
& \frac{S I_{s}}{I_{m}}-\frac{S^{*} I_{s}^{*}}{I_{m}^{*}}=S I_{s}\left(\frac{1}{I_{m}}-\frac{1}{I_{m}^{*}}\right)+\frac{S}{I_{m}^{*}}\left(I_{s}-I_{s}^{*}\right)+\frac{I_{s}^{*}}{I_{m}^{*}}\left(S-S^{*}\right), \\
& \frac{S I_{m}}{I_{s}}-\frac{S^{*} I_{m}^{*}}{I_{s}^{*}}=S I_{m}\left(\frac{1}{I_{s}}-\frac{1}{I_{s}^{*}}\right)+\frac{S}{I_{s}^{*}}\left(I_{m}-I_{m}^{*}\right)+\frac{I_{m}^{*}}{I_{s}^{*}}\left(S-S^{*}\right) .
\end{aligned}
$$

Hence we obtain

$$
\begin{aligned}
\dot{L}= & -\frac{d}{S S^{*}}\left(S-S^{*}\right)^{2} \\
& +\left(S-S^{*}\right)\left(I_{m}-I_{m}^{*}\right)\left[-\beta_{m}+\mu f\left(\beta_{m}+\beta_{s} \frac{I_{s}^{*}}{I_{m}^{*}}\right)\right] \\
& +\left(S-S^{*}\right)\left(I_{s}-I_{s}^{*}\right)\left[-\beta_{s}+\nu(1-f)\left(\beta_{s}+\beta_{m} \frac{I_{m}^{*}}{I_{s}^{*}}\right)\right] \\
& -\mu f \beta_{s} \frac{S I_{s}}{I_{m} I_{m}^{*}}\left(I_{m}-I_{m}^{*}\right)^{2}+\left[\mu f \beta_{s} \frac{S}{I_{m}^{*}}+\nu(1-f) \beta_{m} \frac{S}{I_{s}^{*}}\right]\left(I_{m}-I_{m}^{*}\right)\left(I_{s}-I_{s}^{*}\right) \\
& -\nu(1-f) \beta_{m} \frac{S I_{m}}{I_{s} I_{s}^{*}}\left(I_{s}-I_{s}^{*}\right)^{2} .
\end{aligned}
$$

Denote the sum of the last two lines of equation (10) by $\Phi$. We choose

$$
\begin{aligned}
& \mu=\frac{\beta_{m}}{f\left(\beta_{m}+\beta_{s} \frac{I_{s}^{*}}{I_{m}^{*}}\right)}=\frac{\beta_{m} I_{m}^{*}}{f\left(\beta_{m} I_{m}^{*}+\beta_{s} I_{s}^{*}\right)}, \\
& \nu=\frac{\beta_{s}}{(1-f)\left(\beta_{s}+\beta_{m} \frac{I_{m}^{*}}{I_{s}^{*}}\right)}=\frac{\beta_{s} I_{s}^{*}}{(1-f)\left(\beta_{s} I_{s}^{*}+\beta_{m} I_{m}^{*}\right)},
\end{aligned}
$$

then

$$
\dot{L}=-\frac{d}{S S^{*}}\left(S-S^{*}\right)^{2}+\Phi,
$$

where

$$
\begin{aligned}
\Phi & =-\frac{\beta_{m} \beta_{s} S}{\left(\beta_{m} I_{m}^{*}+\beta_{s} I_{s}^{*}\right)}\left[\frac{I_{s}}{I_{m}}\left(I_{m}-I_{m}^{*}\right)^{2}-2\left(I_{m}-I_{m}^{*}\right)\left(I_{s}-I_{s}^{*}\right)+\frac{I_{m}}{I_{s}}\left(I_{s}-I_{s}^{*}\right)^{2}\right] \\
& =-\frac{\beta_{m} \beta_{s} S}{\left(\beta_{m} I_{m}^{*}+\beta_{s} I_{s}^{*}\right)}\left[\sqrt{\frac{I_{s}}{I_{m}}}\left(I_{m}-I_{m}^{*}\right)-\sqrt{\frac{I_{m}}{I_{s}}}\left(I_{s}-I_{s}^{*}\right)\right]^{2} .
\end{aligned}
$$


Hence $\dot{L} \leq 0$. In the set

$$
M=\left\{\left(S, I_{m}, I_{s}\right): \dot{L}=0\right\}=\left\{S=S^{*}, \sqrt{\frac{I_{s}}{I_{m}}}\left(I_{m}-I_{m}^{*}\right)=\sqrt{\frac{I_{m}}{I_{s}}}\left(I_{s}-I_{s}^{*}\right)\right\},
$$

the maximal invariant set of $M$ is $\left\{E^{*}\right\}$. By LaSalle's invariance principle, $E^{*}$ is globally stable if $\mathcal{R}_{0}>1$.

Finally, consider the boundary cases where $f=0$ or $f=1$. When $f=0$, we choose $\mu=0, \nu=\frac{\beta_{s}}{\gamma_{s}+d}$ for case (a) and $\mu=0, \nu=1$ for case (b). Similarly, when $f=1$ we choose $\mu=\frac{\beta_{m}}{\gamma_{m}+d}, \nu=0$ for case (a) and $\mu=1, \nu=0$ for case (b).

\section{Trade-offs and the basic reproduction number $\mathcal{R}_{0}$}

In this section we consider how the contact-transmissibility trade-off affects system (1) through the transmission parameters $\beta_{m}$ and $\beta_{s}$, which correspondingly affect $\mathcal{R}_{0}$ (cf. (3)). Throughout this section we assume that $\beta(p)=c(p) p$, with the contact rate $c(p)$ satisfying $c^{\prime}(p) \leq 0$ (i.e. contact rate is a monotone decreasing function of $p$ ), where $/$ denotes the derivative. In the special case where $c^{\prime}(p) \equiv 0$, the contact rate is independent of $p$ and $\mathcal{R}_{0}$ is a monotone increasing function of $p$. We exclude this case in the remainder of the section.

From the formula of basic reproduction number (3) and the transmission parameters $(2)$, we regard $\mathcal{R}_{0}$ as a function of $p$, with derivative

$$
R_{0}^{\prime}(p)=\frac{f}{\gamma_{m}+d} \beta_{m}^{\prime}(p)+\frac{1-f}{\gamma_{s}+d} \beta_{s}^{\prime}(p) .
$$

Note that, from (2), we have $\mathcal{R}_{0}^{\prime}(0)=\left[\frac{f}{\gamma_{m}+d} \sigma+\frac{1-f}{\gamma_{s}+d}\right] c(0)>0$. By continuity, $\mathcal{R}_{0}^{\prime}(p)>0$ for $p>0$ sufficiently small. For larger values of $p$ we have to consider all the terms of equation (3). Because $\mathcal{R}_{0}$ is a linear combination of two special cases $f=0$ (severe infections only) and $f=1$ (mild infections only; cf. (3)), we consider them separately.

$$
\begin{aligned}
\text { For } f=0, \mathcal{R}_{0}(p)= & \mathcal{R}_{00}(p) \text { and } \mathcal{R}_{00}^{\prime}(p)=\frac{1}{\gamma_{s}+d}\left(c(p)+c^{\prime}(p) p\right) . \text { Thus } \\
& \mathcal{R}_{00}^{\prime}(p)>0 \text { if } c(p)>p\left|c^{\prime}(p)\right| .
\end{aligned}
$$

Similarly, for $f=1, \mathcal{R}_{0}(p)=\mathcal{R}_{01}(p)$, we have that

$$
\mathcal{R}_{01}^{\prime}(p)>0 \text { if } c(\sigma p)>\sigma p\left|c^{\prime}(\sigma p)\right| .
$$


This gives sufficient criteria for $\mathcal{R}_{0}$ to be increasing (decreasing) for $f \in$ $(0,1)$ :

$$
\begin{array}{ll}
\mathcal{R}_{0}^{\prime}(p)>0 & \text { if } c(p)>p\left|c^{\prime}(p)\right| \text { and } c(\sigma p)>\sigma p\left|c^{\prime}(\sigma p)\right|, \\
\mathcal{R}_{0}^{\prime}(p)<0 & \text { if } c(p)<p\left|c^{\prime}(p)\right| \text { and } c(\sigma p)<\sigma p\left|c^{\prime}(\sigma p)\right| .
\end{array}
$$

Together with the fact that $\mathcal{R}_{0}^{\prime}(0)>0$, the criteria in $(16)$ for $\mathcal{R}_{0}^{\prime}(p)<0$ thus correspond to $\mathcal{R}_{0}$ being non-monotone. These criteria have intuitive biological interpretations: first, that contact rate be sufficiently sensitive to symptom severity $\left(\left|c^{\prime}(p)\right|>c(p) / p\right)$, and second, that mildly symptomatic individuals be sufficiently infectious $\left(\sigma>c(\sigma p) / p\left|c^{\prime}(\sigma p)\right|\right)$.

Determining the shape of $\mathcal{R}_{0}(p)$ when (16) does not hold is more difficult. We focus on the simple case where $\beta(p)$ satisfies the following assumptions: (A1) there exists a unique $p^{*}$ such that $\beta(p)$ first increases to maximum $\beta\left(p^{*}\right)$ then decays; (A2) there exists at most a single inflection point on $\left(p^{*}, \infty\right)$. Let $p^{* *}$ denote the inflection point (if it exists). We assume that $\beta(p)$ is concave down on $\left(p^{*}, p^{* *}\right)$ and concave up on $\left(p^{* *}, \infty\right)$. Note that these assumptions ensure that $\beta(p)$ has only one local maximum.

Let $q=\sigma p$. From $(2), \beta_{m}(p)=\beta_{s}(q)$ has a unique critical point $q^{*}=p^{*}$ (the graph of $\beta_{m}(p)$ matches that of $\beta_{s}(p)$ after scaling the domain by $1 / \sigma$ ). From equation (16), we know that $\mathcal{R}_{0}^{\prime}(p)>0$ for $p \leq p^{*} ; \mathcal{R}_{0}^{\prime}(p)<0$ for $p \geq \frac{p^{*}}{\sigma}$. Thus, critical points of $\mathcal{R}_{0}(p)$ are located in the interval $\left(p^{*}, \frac{p^{*}}{\sigma}\right)$ and satisfy

$$
d R_{+}:=\frac{f}{\gamma_{m}+d} \beta_{m}^{\prime}(p)=-\frac{1-f}{\gamma_{s}+d} \beta_{s}^{\prime}(p)=: d R_{-} .
$$

The number of critical points of $\mathcal{R}_{0}(p)$ are determined by the relationship between $\frac{p^{*}}{\sigma}$ and $p^{* *}$.

For the case $p^{*}<\frac{p^{*}}{\sigma}<p^{* *}$, the function $d R_{+}$is positive and strictly decreasing on $\left(p^{*}, \frac{p^{*}}{\sigma}\right)$, with $d R_{+}\left(\frac{p^{*}}{\sigma}\right)=0$. On the other hand, $d R_{-}$is strictly increasing on $\left(p^{*}, \frac{p^{*}}{\sigma}\right)$ and $d R_{-}\left(p^{*}\right)=0$. By the intermediate value theorem, there exists a unique $\bar{p}$ such that (17) holds.

For the case $p^{*}<p^{* *}<\frac{p^{*}}{\sigma}$, the function $d R_{+}$keeps the same property as

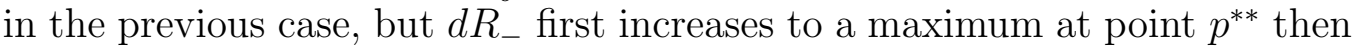
decays. From the graph below, we can see there are at most three intersection points of $d R_{+}$and $d R_{-}$.

Whether $\beta(p)$ satisfies assumptions (A1) and (A2) depends upon the shape of $c(p)$. A sufficient condition for (A1) and (A2) to hold is for the twice differentiable $c(p)$ to have $c^{\prime \prime}(p)<0$ on $(0,1)$. The critical point of 


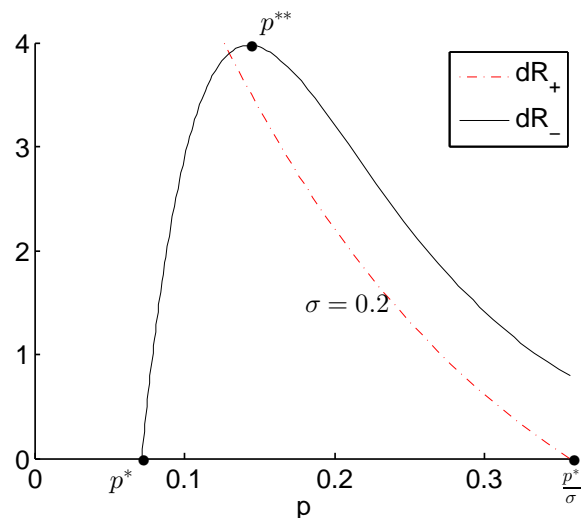

(a)

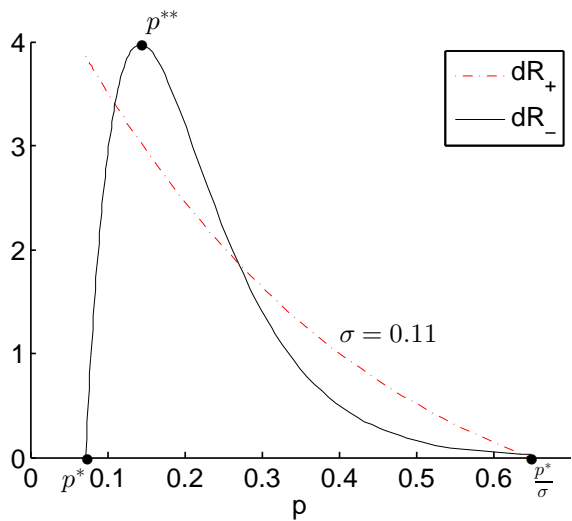

(b)

Figure 2: Illustration of the number of critical points of $\mathcal{R}_{0}$ for two different values of $\sigma$, corresponding to intersections of $d R_{+}$and $d R_{-}$. Here $c(p)=a e^{-\lambda p}$, with $a=11.1$, $\lambda=14, \gamma_{m}=\gamma_{s}=\frac{1}{7}, f=0.6, d=0.0083$.

$\beta(p)$ satisfies

$$
p^{*}=\frac{c\left(p^{*}\right)}{-c^{\prime}\left(p^{*}\right)} .
$$

That is, $p^{*}$ is the intersection of $y=p$ and $y=-c(p) / c^{\prime}(p)$ which is infinity at points $p$ satisfying $c^{\prime}(p)=0$.

If $c^{\prime \prime}(p)<0$, we have

$$
\frac{d}{d p}\left(\frac{c(p)}{-c^{\prime}(p)}\right)=-\frac{\left(c^{\prime}(p)\right)^{2}-c(p) c^{\prime \prime}(p)}{\left(c^{\prime}(p)\right)^{2}}<0,
$$

and there is a unique $p^{*}$ that satisfies (18). From the fact

$$
\beta^{\prime \prime}(p)=2 c^{\prime}(p)+p c^{\prime \prime}(p)<0,
$$

we know that $\beta(p)$ is concave down for all $p$ and there is a unique critical point of $\mathcal{R}_{0}(p)$.

The case where $c(p)$ is not always concave down is more complicated. In the absence of empirical data on the form of $c(p)$, for simplicity we focus on the special case where contact rates decay exponentially with $p$ :

$$
c(p):=a e^{-\lambda p}
$$

where $a$ is the baseline contact rate in the absence of infection, and $\lambda$ the decay rate determining how rapidly contact rates decrease with symptom 
severity. Note that $c^{\prime \prime}(p)>0$ in this case. For $(19), \beta_{s}(p)$ first increases to the maximum $\beta_{s}(1 / \lambda)$ and then decreases, and here $p^{*}=1 / \lambda$. Note that when $\lambda \leq 1$, then both $\beta_{s}(p)$ and $\beta_{m}(p)$ are increasing in $p$ for all $p \in[0,1]$, and thus $\mathcal{R}_{0}$ is increasing on $[0,1]$ as well. The following lemma shows that when $\lambda>1, \mathcal{R}_{0}(p)$ has at most three critical points.

Lemma 1. Suppose $\lambda>1$. There is a unique critical point of $\mathcal{R}_{0}(p)$ if either scenarios (a) or (b) below holds:

(a) $\sigma \geq 3-2 \sqrt{2}$;

(b) $\sigma<3-2 \sqrt{2}$, and either $y>y_{-}(\sigma)$ or $y<y_{+}(\sigma)$ where $y=\frac{f}{1-f} \frac{\gamma_{s}+d}{\gamma_{m}+d}$.

The functions $y_{ \pm}(\sigma)$ are defined by

$$
y_{ \pm}(\sigma):=\frac{1}{\sigma} e^{-x_{ \pm}(1-\sigma)}\left(x_{ \pm}-1\right)^{2}
$$

where

$$
x_{ \pm}:=\frac{1+\sigma \pm \sqrt{\sigma^{2}-6 \sigma+1}}{2 \sigma} .
$$

When $\sigma<3-2 \sqrt{2}$ and $y_{+}(\sigma)<y<y_{-}(\sigma)$, then there are three critical points of $\mathcal{R}_{0}(p)$.

Proof. Any critical point $\bar{p}$ of $\mathcal{R}_{0}$ satisfies (17) and lies in the interval $\left(\frac{1}{\lambda}, \frac{1}{\lambda \sigma}\right)$, where $\beta_{s}(p)$ is decreasing and $\beta_{m}(p)$ is increasing in $p$. From (17), we obtain that

$$
\frac{f \sigma}{1-f} \frac{\gamma_{s}+d}{\gamma_{m}+d} e^{\lambda \bar{p}(1-\sigma)}=\frac{\lambda \bar{p}-1}{1-\lambda \sigma \bar{p}}
$$

and

$$
\ln \left(\frac{f \sigma}{1-f} \frac{\gamma_{s}+d}{\gamma_{m}+d}\right)+\lambda \bar{p}(1-\sigma)=\ln (\lambda \bar{p}-1)-\ln (1-\lambda \sigma \bar{p}) .
$$

Let $x=\lambda p$ and $y=\frac{f}{1-f} \frac{\gamma_{s}+d}{\gamma_{m}+d}$. Define two functions of $x$ on the interval $\left(1, \frac{1}{\sigma}\right)$ by

$$
\begin{aligned}
& H_{1}(x)=\ln (y \sigma)+x(1-\sigma), \\
& H_{2}(x)=\ln (x-1)-\ln (1-\sigma x) .
\end{aligned}
$$

Then the critical points of $\mathcal{R}_{0}$ satisfy $\bar{p}=\bar{x} / \lambda$, where $\bar{x}$ are the intersections of $H_{1}(x)$ and $H_{2}(x)$. Note that $H_{1}$ is a straight line. Direct computation 
gives

$$
\begin{aligned}
& H_{2}^{\prime}(x)=\frac{1-\sigma}{(1-\sigma x)(x-1)}>0 \\
& H_{2}^{\prime \prime}(x)=\frac{-(1+\sigma-2 \sigma x)}{(1-\sigma x)^{2}(x-1)^{2}} .
\end{aligned}
$$

Thus $H_{2}$ is concave down on $\left(1, \frac{1}{2}+\frac{1}{2 \sigma}\right)$, and concave up on $\left(\frac{1}{2}+\frac{1}{2 \sigma}, \frac{1}{\sigma}\right)$.

Note that $H_{2}\left(1^{+}\right)=-\infty<H_{1}(1)$, and $H_{2}\left(\frac{1}{\sigma}^{-}\right)=\infty>H_{1}\left(\frac{1}{\sigma}\right)$. By the intermediate value theorem, there exists $\bar{x}$ such that $H_{1}(\bar{x})=H_{2}(\bar{x})$. If there are more than one intersection points, by the mean value theorem there exists at least one point where $H_{2}^{\prime}(x)=H_{1}^{\prime}(x)$.

Setting $H_{1}^{\prime}(x)=H_{2}^{\prime}(x)$ gives

$$
(1-\sigma x)(x-1)=1
$$

or

$$
x_{ \pm}(\sigma):=\frac{1+\sigma \pm \sqrt{\Delta}}{2 \sigma},
$$

where $\Delta=\sigma^{2}-6 \sigma+1$. Note that $\Delta \geq 0$ if $\sigma \leq 3-2 \sqrt{2} \approx 0.1716$. Also it is clear that

$$
H_{1}^{\prime}(x)>H_{2}^{\prime}(x) \text { for all } x \in\left(x_{-}, x_{+}\right) .
$$

If $\sigma>3-2 \sqrt{2}$, then there is no real $x_{ \pm}$satisfying $H_{2}^{\prime}(x)=H_{1}^{\prime}(x)$. Thus there is only one intersection point of $H_{1}(x)$ and $H_{2}(x)$.

If $\sigma \leq 3-2 \sqrt{2}$, then we have $0<x_{-} \leq x_{+}$. Hence there are at most three intersection points, with the number depending on the sign of $\left(H_{1}\left(x_{ \pm}\right)-\right.$ $\left.H_{2}\left(x_{ \pm}\right)\right)$. Let $y_{ \pm}(\sigma)$ denote the values for $y$ where $H_{1}\left(x_{ \pm}\right)=H_{2}\left(x_{ \pm}\right)$. This gives

$$
\begin{aligned}
y_{ \pm} \sigma e^{x_{ \pm}(1-\sigma)} & =\frac{x_{ \pm}-1}{1-\sigma x_{ \pm}} \\
& =\left(x_{ \pm}-1\right)^{2},
\end{aligned}
$$

with the last equality following from equation (25). For any fixed parameter $y$, the function $H_{1}(x)=H_{1}(x ; y)$ is linear in $x$ with slope independent of $y$. From $(27)$ and $H_{1}\left(x_{ \pm} ; y_{ \pm}\right)=H_{2}\left(x_{ \pm}\right)$, we have

$$
H_{1}\left(x_{+} ; y_{-}\right)>H_{2}\left(x_{+}\right)=H_{1}\left(x_{+} ; y_{+}\right)
$$

Thus

$$
0<H_{1}\left(x ; y_{-}\right)-H_{1}\left(x ; y_{+}\right)=\ln \left(y_{-} \sigma\right)-\ln \left(y_{+} \sigma\right)=\ln \frac{y_{-}}{y_{+}}
$$


and $y_{-}>y_{+}$.

When $y \in\left(y_{+}, y_{-}\right)$, which means $H_{1}\left(x_{-}\right)<H_{2}\left(x_{-}\right)$and $H_{1}\left(x_{+}\right)>$ $H_{2}\left(x_{+}\right)$, there are three intersection points of $H_{1}$ and $H_{2}$. Hence there is only one intersection point when $y \notin\left[y_{+}, y_{-}\right]$.

Lemma 1 thus describes how the $(y, \sigma)$-parameter plane is divided into regions with one versus three critical points of $\mathcal{R}_{0}(p)$, with the dividing boundaries corresponding to two critical points. Provided that $\lambda>1$, the locations of the dividing boundaries do not depend upon the specific value of $\lambda$, nor upon the value of the baseline contact rate $a$. These regions are illustrated in Figure 3.

Note that multiple critical points require intermediate values of $y$ (specifically, $\left.y_{+}(\sigma)<y<y_{-}(\sigma)\right)$. This is a biologically intuitive result, as $y$ can be expressed as the ratio of a measure of infectivity of the mild $\left(f /\left(\gamma_{m}+d\right)\right)$ and severe $\left((1-f) /\left(\gamma_{s}+d\right)\right)$ compartments. For intermediate values of this ratio, the contribution of both the mild and severe compartments to infection is significant, and we have local maxima of $\mathcal{R}_{0}$ at high and low values of $p$.

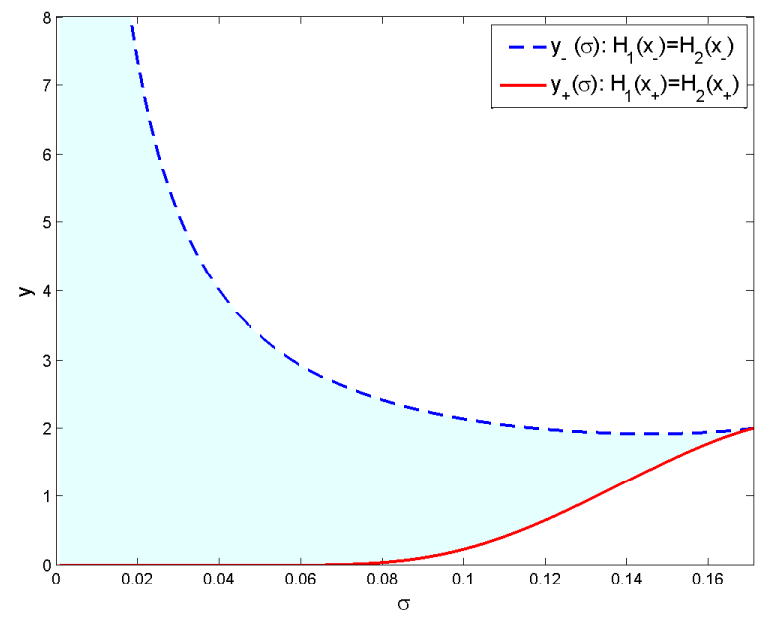

Figure 3: Illustration of the number of critical points of $\mathcal{R}_{0}$ in the $(\sigma, y)$-plane, where $\sigma$ is the relative transmissibility of mild infections and $y=\frac{f}{1-f} \frac{\gamma_{s}+d}{\gamma_{m}+d}$, when contact rate satisfies $c(p)=a e^{-\lambda p}, \lambda>1$. Shaded region corresponds to three critical points, white region corresponds to one critical point.

Of course, $p$ is a probability, and thus we are only interested in critical points corresponding to $p$ in $(0,1)$. An example where $\mathcal{R}_{0}(p)$ has three critical 
points in $(0,1)$ is shown in Figure 4.

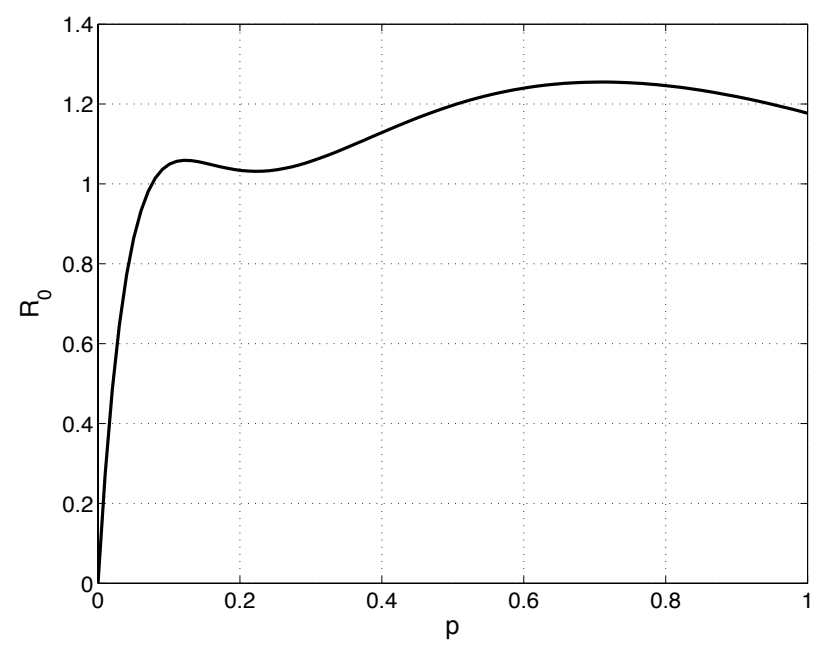

Figure 4: An example where $\mathcal{R}_{0}(p)$ has three critical points. Contact function $c(p)=$ $a \exp (-\lambda p)$, with parameter values $a=11.1, \lambda=14, f=0.65, d=0.0083, \gamma_{s}=\gamma_{m}=1 / 7$, $\sigma=0.1$.

\section{Competitive exclusion and strain selection}

\subsection{Competitive exclusion}

To consider the evolutionary implications of the trade-off between contact and transmissibility, consider a multi-strain version of system (1). Strains may differ in their symptom severity (and thus in their transmission coefficients $\beta_{i}$ ), as well as in their recovery rates, and proportion of mild and severe infections. Correspondingly, the strains may possess different reproduction numbers $\mathcal{R}_{0}^{i}$. Additionally, we assume that susceptible hosts can only be infected by a single strain (i.e. no super-infection), and that recovery following infection by any strain confers infection-derived immunity to all other strains. These assumptions are the same as those made by Bremermann and Thieme [6], in their investigations of how the trade-off between transmissibility and disease-induced mortality affects pathogen virulence evolution. One of Bremermann and Thieme's main findings is that under these assumptions, the pathogen strain with highest basic reproduction number drives the other strains to extinction. In this section, we similarly show competitive exclusion by the highest $\mathcal{R}_{0}$ strain for the multi-strain version of (1). 
$S \quad$ The proportion of susceptible individuals

$I_{i}^{m} \quad$ The proportion of mildly infected individuals of the $i$ th strain

$I_{i}^{s} \quad$ The proportion of severely infected individuals of the $i$ th strain

$d \quad$ Birth rate and natural death rate

$\beta_{i}^{m} \quad$ Transmission parameters for the $i$ th strain mild infection

$\beta_{i}^{s} \quad$ Transmission parameters for the $i$ th strain severe infection

$f_{i} \quad$ Fraction of mild infection of the $i$ th strain

$\gamma_{i}^{m} \quad$ Recovery rate of the $i$ th strain mild infection

$\gamma_{i}^{s} \quad$ Recovery rate of the $i$ th strain severe infection

Table 1: Model variables and parameters

The multi-strain model equations are given in system (29):

$$
\begin{aligned}
& \dot{S}=d-d S-\sum_{i=1}^{n} S\left(\beta_{i}^{m} I_{i}^{m}+\beta_{i}^{s} I_{i}^{s}\right), \\
& \dot{I}_{i}^{m}=f_{i} S\left(\beta_{i}^{m} I_{i}^{m}+\beta_{i}^{s} I_{i}^{s}\right)-\left(\gamma_{i}^{m}+d\right) I_{i}^{m} \\
& \dot{I}_{i}^{s}=\left(1-f_{i}\right) S\left(\beta_{i}^{m} I_{i}^{m}+\beta_{i}^{s} I_{i}^{s}\right)-\left(\gamma_{i}^{s}+d\right) I_{i}^{s},
\end{aligned}
$$

where the subscripts $i=1,2, \ldots, n$ denote the pathogen strain. Variables and parameters for the multi-strain model are summarized in Table 1. The total population $N=S+\sum_{i=1}^{n}\left(I_{i}^{m}+I_{i}^{s}\right)+R$ also equals to 1 , and all variables are fractions of the population.

The basic reproduction number for strain $i$ is

$$
\mathcal{R}_{0}^{i}=f_{i} \frac{\beta_{i}^{m}}{\gamma_{i}^{m}+d}+\left(1-f_{i}\right) \frac{\beta_{i}^{s}}{\gamma_{i}^{s}+d}
$$

When $\mathcal{R}_{0}^{i} \leq 1$ for all $i$, the disease-free equilibrium $E_{0}$ is globally stable and all pathogen strains go to extinction by adapting the similar Lyapunov function in (5). When $\mathcal{R}_{0}^{i}>1$ for some strain $i$, there exists an exclusion equilibrium $E_{i}$ where strain $i$ persists and all other strains are absent:

$$
S_{i}^{*}=\frac{1}{\mathcal{R}_{0}^{i}}, I_{i}^{m *}=\frac{f_{i}}{\gamma_{i}^{m}+d} d\left(1-\frac{1}{\mathcal{R}_{0}^{i}}\right), I_{i}^{s *}=\frac{1-f_{i}}{\gamma_{i}^{s}+d} d\left(1-\frac{1}{\mathcal{R}_{0}^{i}}\right),
$$

and $I_{j}^{m}=I_{j}^{s}=0$ for $j \neq i$. 
In considering whether coexistence is possible for system (29), we restrict attention to the generic case where all strains have distinct $\mathcal{R}_{0}^{i}$ from one another. Note also that if $\mathcal{R}_{0}^{i} \leq 1$ for some $i$, by adapting the same Lyapunov function in (5), we know that strain $i$ will go to extinction. We thus only consider the case where $\mathcal{R}_{0}^{i}>1$ for all strains. Theorem 2 shows that in this situation, coexistence in system (29) does not occur.

Theorem 2. Suppose $d>0, \beta_{i}^{m}, \beta_{i}^{s} \geq 0, \gamma_{i}^{m}, \gamma_{i}^{s}>0$ and $f_{i} \in[0,1]$. Assume $\mathcal{R}_{0}^{1}>\mathcal{R}_{0}^{i}>1$ for all $i \neq 1$, then $E_{1}$ is globally stable for all initial condition in $\Omega_{n}$, where

$$
\Omega_{n}:=\left\{\left(S, I_{1}^{m}, I_{1}^{s}, \ldots, I_{n}^{m}, I_{n}^{s}\right) \in[0,1]^{2 n+1}: S>0, S+\sum_{i=1}^{n}\left(I_{i}^{m}+I_{i}^{s}\right) \leq 1\right\} .
$$

Proof. We first consider that case where $f_{i} \in(0,1)$ for all $i$. Let

$L(t)=\int_{S_{1}^{*}}^{S} \frac{\eta-S_{1}^{*}}{\eta} d \eta+\mu_{1} \int_{I_{1}^{m *}}^{I_{1}^{m}} \frac{\eta-I_{1}^{m *}}{\eta} d \eta+\nu_{1} \int_{I_{1}^{s *}}^{I_{1}^{s}} \frac{\eta-I_{1}^{s *}}{\eta} d \eta+\sum_{i=2}^{n}\left(\mu_{i} I_{i}^{m}+\nu_{i} I_{i}^{s}\right)$,

where $\mu_{i}, \nu_{i}$ are to be determined later for $i=1,2, \ldots, n$. Then

$$
\dot{L}=L_{1}\left(S, I_{1}^{m}, I_{1}^{s}\right)+\sum_{i=2}^{n} L_{i}\left(S, I_{i}^{m}, I_{i}^{s}\right),
$$

where $L_{1}\left(S, I_{1}^{m}, I_{1}^{s}\right)$ has the same form as (10). By choosing

$$
\mu_{1}=\frac{\beta_{1}^{m} I_{1}^{m *}}{f_{1}\left(\beta_{1}^{m} I_{1}^{m *}+\beta_{1}^{s} I_{1}^{s *}\right)}, \nu_{1}=\frac{\beta_{1}^{s} I_{1}^{s *}}{\left(1-f_{1}\right)\left(\beta_{1}^{s} I_{1}^{s *}+\beta_{1}^{m} I_{1}^{m *}\right)},
$$

we obtain $L_{1}\left(S, I_{1}^{m}, I_{1}^{s}\right) \leq 0$. For $i \geq 2$,

$$
\begin{aligned}
L_{i}\left(S, I_{i}^{m}, I_{i}^{s}\right)= & -\left(S-S_{1}^{*}\right)\left(\beta_{i}^{m} I_{i}^{m}+\beta_{i}^{s} I_{i}^{s}\right) \\
& +\mu_{i}\left[f_{i} S\left(\beta_{i}^{m} I_{i}^{m}+\beta_{i}^{s} I_{i}^{s}\right)-\left(\gamma_{i}^{m}+d\right) I_{i}^{m}\right] \\
& +\nu_{i}\left[\left(1-f_{i}\right) S\left(\beta_{i}^{m} I_{i}^{m}+\beta_{i}^{s} I_{i}^{s}\right)-\left(\gamma_{i}^{s}+d\right) I_{i}^{s}\right] \\
= & S\left(\beta_{i}^{m} I_{i}^{m}+\beta_{i}^{s} I_{i}^{s}\right)\left(-1+\mu_{i} f_{i}+\nu_{i}\left(1-f_{i}\right)\right) \\
& +I_{i}^{m}\left(\beta_{i}^{m} S_{1}^{*}-\mu_{i}\left(\gamma_{i}^{m}+d\right)\right)+I_{i}^{s}\left(\beta_{i}^{s} S_{1}^{*}-\nu_{i}\left(\gamma_{i}^{s}+d\right)\right) .
\end{aligned}
$$


Let $\mu_{i}=\frac{1}{\mathcal{R}_{0}^{1}} \frac{\beta_{i}^{m}}{\gamma_{i}^{m}+d}, \nu_{i}=\frac{1}{\mathcal{R}_{0}^{1}} \frac{\beta_{i}^{s}}{\gamma_{i}^{s}+d}$. Then

$$
\begin{aligned}
-1+\mu_{i} f_{i}+\nu_{i}\left(1-f_{i}\right) & =-1+\frac{1}{\mathcal{R}_{0}^{1}}\left(f_{i} \frac{\beta_{i}^{m}}{\gamma_{i}^{m}+d}+\left(1-f_{i}\right) \frac{\beta_{i}^{s}}{\gamma_{i}^{s}+d}\right) \\
=-1+\frac{\mathcal{R}_{0}^{i}}{\mathcal{R}_{0}^{1}}<0, & < \\
\beta_{i}^{m} S_{1}^{*}-\mu_{i}\left(\gamma_{i}^{m}+d\right) & =\beta_{i}^{m} \frac{1}{\mathcal{R}_{0}^{1}}-\frac{1}{\mathcal{R}_{0}^{1}} \beta_{i}^{m} \\
& =0, \\
\beta_{i}^{s} S_{1}^{*}-\nu_{i}\left(\gamma_{i}^{s}+d\right) & =0 .
\end{aligned}
$$

This gives $L_{i}\left(S, I_{i}^{m}, I_{i}^{s}\right) \leq 0$. Now we conclude that $\dot{L}(t) \leq 0$. In the set

$$
\begin{aligned}
M & =\left\{\left(S, I_{1}^{m}, I_{1}^{s}, \ldots, I_{n}^{m}, I_{n}^{s}\right): \dot{L}=0\right\} \\
& =\left\{S=S_{1}^{*}, \sqrt{\frac{I_{1}^{s}}{I_{1}^{m}}}\left(I_{1}^{m}-I_{1}^{m *}\right)=\sqrt{\frac{I_{1}^{m}}{I_{1}^{s}}}\left(I_{1}^{s}-I_{1}^{s *}\right), I_{i}^{m}=I_{i}^{s}=0, \text { for } i=2,3, \ldots, n\right\},
\end{aligned}
$$

the maximal invariant set of $M$ is $\left\{E_{1}\right\}$. From the LaSalle's invariance principle, $E_{1}$ is globally stable.

Finally, for the cases $f_{i}=0$ and $f_{i}=1$ for some $i$, we choose $\mu_{i}=0, \nu_{i}=1$ and $\mu_{i}=1, \nu_{i}=0$, respectively.

\subsection{Adaptive dynamics}

Theorem 2 states that strain competition will result in competitive exclusion by the highest $\mathcal{R}_{0}$ strain. This 'ecological' result has evolutionary implications, suggesting selection for $\mathcal{R}_{0}$ maximization in system (29). In this section we study $\mathcal{R}_{0}$ selection using adaptive dynamics, a set of theoretical tools developed in the last twenty five years ([37] section 2.1) to examine "evolutionary changes in the values of traits of representative individuals" [24]. In particular, adaptive dynamics provides a set of tools to link the ecological and evolutionary timescales to look at evolutionary outcomes [10]. Here we examine adaptive dynamics in the setting where $\mathcal{R}_{0}$ has more than one local maxima as a function of the probability of transmission $p$.

We focus in this section on selection on the transmission probability $p$, where contact rate $c(p)$ is linked to $p$ through the contact-transmissibility trade-off. Let $p_{1}$ denote the transmission probability of the resident strain, and $p_{2}$ the transmission probability of a mutant strain. Whether the mutant 
is able to successfully invade the population depends upon the invasion exponent, representing the fitness of the mutant in the environment established by the resident [10]. Let $r_{p_{1}}\left(p_{2}\right)$ denote the invasion exponent of a mutant strain with trait $p_{2}$, amongst a resident population with trait $p_{1}$. Here we take the invasion exponent to be

$$
r_{p_{1}}\left(p_{2}\right)=\mathcal{R}_{0}\left(p_{2}\right) S\left(p_{1}\right)-1,
$$

where $S\left(p_{1}\right)$ is the number of susceptible individuals at the endemic equilibrium consisting of the resident strain only. Thus $\mathcal{R}_{0}\left(p_{2}\right) S\left(p_{1}\right)$ is the reproduction number of the mutant strain in the environment established by the resident, and the invasion exponent is positive when the reproduction number is larger than one. Equation (35) can equivalently be derived as a weighted per capita growth rate of the infected compartments:

$$
r_{p_{1}}\left(p_{2}\right)=\frac{\frac{d}{d t}\left(\frac{\beta_{m}}{\gamma_{m}+d} I_{m}+\frac{\beta_{s}}{\gamma_{s}+d} I_{s}\right)}{\beta_{m} I_{m}+\beta_{s} I_{s}} .
$$

As $S\left(p_{1}\right)=1 / \mathcal{R}_{0}\left(p_{1}\right),(35)$ becomes

$$
r_{p_{1}}\left(p_{2}\right)=\mathcal{R}_{0}\left(p_{2}\right) \mathcal{R}_{0}^{-1}\left(p_{1}\right)-1 .
$$

Differentiating (37) with respect to $p_{2}$ gives the selection gradient:

$$
\left.\frac{\partial}{\partial p_{2}} r_{p_{1}}\left(p_{2}\right)\right|_{p_{2}=p_{1}}=\mathcal{R}_{0}^{\prime}\left(p_{1}\right) \mathcal{R}_{0}^{-1}\left(p_{1}\right) .
$$

The sign of the selection gradient determines whether nearby mutants can invade, with evolution in the direction of the selection gradient [16]. Singular strategies correspond to points where the selection gradient vanishes. Let $\bar{p}$ denote a singular strategy. From (38), $\bar{p}$ satisfies $\mathcal{R}_{0}^{\prime}(\bar{p})=0$, and thus singular strategies correspond to critical points of $\mathcal{R}_{0}(p)$. Following the classification of singular strategies given in Diekmann [10], let

$$
\begin{gathered}
a(\bar{p})=\left.\frac{\partial^{2}}{\partial p_{1}^{2}} r_{p_{1}}\left(p_{2}\right)\right|_{p_{2}=p_{1}=\bar{p}}=-\mathcal{R}_{0}^{\prime \prime}(\bar{p}) \mathcal{R}_{0}^{-1}(\bar{p}), \\
b(\bar{p})=\left.\frac{\partial^{2}}{\partial p_{2}^{2}} r_{p_{1}}\left(p_{2}\right)\right|_{p_{2}=p_{1}=\bar{p}}=\mathcal{R}_{0}^{\prime \prime}(\bar{p}) \mathcal{R}_{0}^{-1}(\bar{p}) .
\end{gathered}
$$

An evolutionarily stable strategy (ESS) corresponds to a strategy that is non-invasible when it is the resident, and a convergence stable strategy is 
one that attracts nearby strategies. A repeller is a singular strategy from which nearby strategies diverge. A continuously stable strategy (CSS) is a convergence ESS. As described in Diekmann [10], a singular strategy is a (local) ESS if $b(\bar{p})<0$, is a convergence stable strategy if $a(\bar{p})>b(\bar{p})$, and is a repeller when $a(\bar{p})<b(\bar{p})$.

Singular strategies correspond here to critical points of $\mathcal{R}_{0}(p)$, with the type of singular strategy determined by the concavity. In particular, local maxima of $\mathcal{R}_{0}(p)$ have $b(\bar{p})<0$, and thus correspond to a local ESS. Local maxima additionally correspond to $a(\bar{p})>0$, so $a(\bar{p})>0>b(\bar{p})$ and local maxima correspond to CSS as well. Local minima have $a(\bar{p})<0<b(\bar{p})$, and thus correspond to repellers.

In cases where $\mathcal{R}_{0}$ has a unique critical point for $p$ in $(0,1)$ (for example, for $c(p)$ concave down, or for exponential $c(p)$ under conditions (a) or (b) of Lemma 1), the critical point corresponds to an ESS. Indeed, Theorem 2 implies that this is a global ESS. In this situation, the model predicts evolution towards intermediate virulence, as a consequence of the transmissibilitycontact trade-off.

The more interesting setting is when $\mathcal{R}_{0}$ has multiple critical points. For example, three critical points exist when neither conditions $(a)$ nor $(b)$ of Lemma 1 are met. Let $\bar{p}_{a 1}<\bar{p}_{r}<\bar{p}_{a 2}$ denote these critical points, where $\bar{p}_{a 1}, \bar{p}_{a 2}$ correspond to local maxima and $\bar{p}_{r}$ is a local minimum. An example is shown in Figure 4, where $\mathcal{R}_{0}$ has a global maximum at $\bar{p}_{a 2}$. Both $\bar{p}_{a 1}$ and $\bar{p}_{a 2}$ are CSS, and thus introduction of mutant strains close to either of these points will lead to convergence to the corresponding local maximum. This is an example of evolutionary bistability, where two continuously stable strategies are separated by a repeller [4]. In particular, this includes convergence of nearby traits to $\bar{p}_{a 1}$, which maximizes $\mathcal{R}_{0}$ locally but not globally. On the other hand, Theorem 2 says that in direct competition, $\bar{p}_{a 2}$ will drive $\bar{p}_{a 1}$ to extinction, suggesting that evolution should eventually drive $p$ to the global maximum $\bar{p}_{a 2}$. However, strategies $\bar{p}_{a 1}$ and $\bar{p}_{a 2}$ may be widely separated in the evolutionary landscape, and a large change in $p$ may be required to escape the basin of attraction of the local maximum $\bar{p}_{a 1}$. Such large mutations may be exceedingly rare. Evolutionary trajectories may thus appear to converge to a local but not global maximum of $\mathcal{R}_{0}$.

Figure 5a shows a pairwise invasibility plot, with resident trait $p_{1}$ on the $x$-axis and mutant trait $p_{2}$ on the $y$-axis. Shaded regions denote locations where the invasion exponent is positive, and white regions where the invasion exponent is negative. The boundaries where the invasion exponent is zero 


\section{(a)}
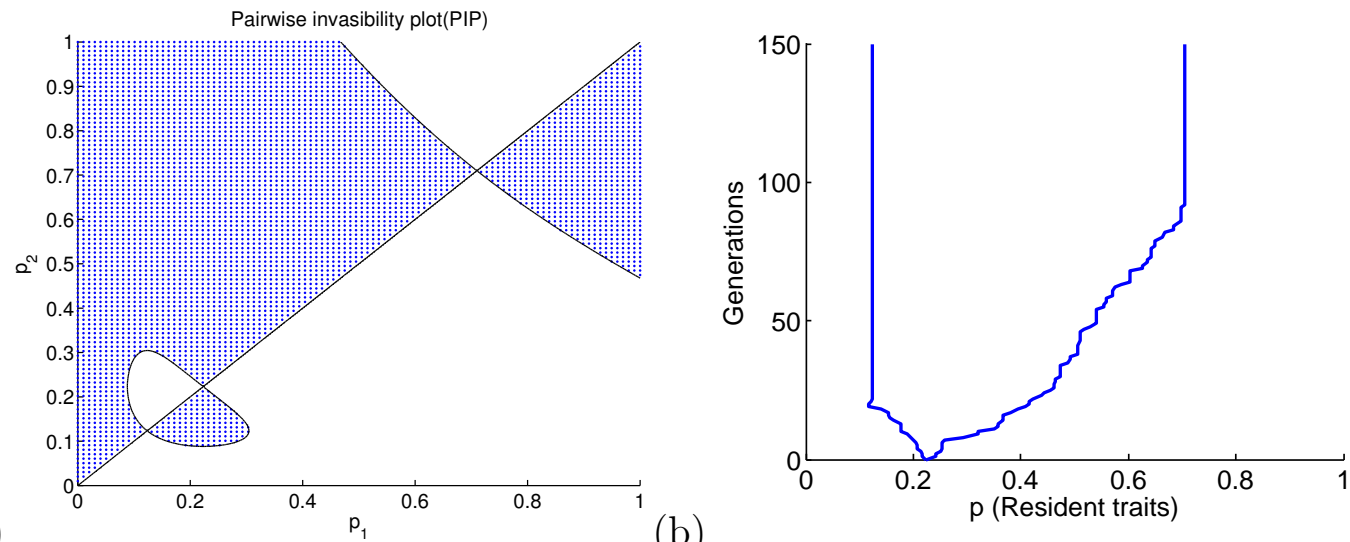

(b)

Figure 5: (a) Pairwise invasibility plot, showing regions where a rare mutant with trait $p_{2}$ can or cannot invade a resident population with trait $p_{1}$. Successful invasion occurs in the shaded regions, where $r_{p_{1}}\left(p_{2}\right)>0$ according to (35). Intersections of the boundary lines separating the shaded and white regions with the $p_{2}=p_{1}$ line correspond to singular strategies. Here there are three singular strategies, at $p_{1}=0.1234$ (local but not global ESS), $p_{1}=0.2231$ (repeller), and $p_{1}=0.7098$ (global ESS). Contact function $c(p)=$ $a \exp (-\lambda p)$, with parameter values $f=0.65, d=0.0083, \gamma_{m}=\gamma_{s}=1 / 7, \sigma=0.1, \lambda=$ 14, $a=11.1$. (b) Two trait trajectories with totally different outcomes starting from the same initial condition $p=0.224$, which is near the repeller strategy.

are shown in solid black lines, and intersections of these boundary lines with the $p_{2}=p_{1}$ line correspond to singular strategies [16]. Three singular strategies exist in Figure 5a, corresponding to $\bar{p}_{a 1}=0.1234$ (local but not global maximum of $\mathcal{R}_{0}$ ), $\bar{p}_{r}=0.2231$ (local minimum of $\mathcal{R}_{0}$ ), and $\bar{p}_{a 2}=0.7098$ (global maximum of $\mathcal{R}_{0}$ ). Note the white region above the singular strategy $\bar{p}$, corresponding to $p_{1}=\bar{p}$ being a local ESS. To successfully invade $\bar{p}$, a non-local mutant trait outside of this white region is required. Generating such a non-local mutant may be a rare event, depending upon the mutation rate and genetic variability in the population.

Figure 5b shows two sample trajectories of the resident trait value over time, using simulations starting from an initial trait value of $p=0.224$, close to the repeller $\bar{p}_{r}$. Following the algorithm of Best and Hoyle [3], mutant traits are generated at random by drawing from a normal distribution centered at the resident trait value. Competition is simulated by numerically computing solution trajectories for model (29) with $n=2$ for a long time span, with initial conditions corresponding to a small mutant population $\left(I_{m}(0)=I_{s}(0)=0.0001\right)$. At the end of the time span, strains with less 
than one infected individual are set to zero, the remaining strains are set as residents for the next iteration, and the process repeats. From Figure 5b, we observe that whether trajectories eventually converge to $\bar{p}_{a 1}$ or $\bar{p}_{a 2}$ depends upon initial fluctuations from the starting point. Additionally, the trajectory shown in Figure 5b converging to $\bar{p}_{a 1}$ remains near $\bar{p}_{a 1}$ for the remainder of the simulation, despite this being only a local maximum for $\mathcal{R}_{0}$.

\section{Discussion}

The impact of illness on contact patterns presents a twist on the classical problem of pathogen virulence evolution. The simple model presented here shows that the inherent trade-off between contact and transmissibility can lead to selection for intermediate levels of virulence, analogous to the classical trade-off between mortality and transmissibility that has been extensively studied $[2,6,7,13,14,15,20]$. Our main mathematical result is that the model exhibits eventual competitive exclusion by the highest $\mathcal{R}_{0}$ strain. We give criteria for when $\mathcal{R}_{0}$ is maximized at intermediate virulence levels, as measured in terms of symptom severity. Indeed, this paper shows that the transmissibility-contact trade-off can result in multiple local maxima for $\mathcal{R}_{0}$ for a model with heterogeneity in disease severity. While the strain with highest $\mathcal{R}_{0}$ will eventually drive the other strains to extinction in the model, transients may be long, with prolonged coexistence between strains. The model presented here is intentionally simple, for example neglecting spatial heterogeneity, differential host susceptibility, and host-pathogen coevolution. Investigating evolutionary and epidemiological outcomes of the contact-transmissibility trade-off in these settings is an important area for future work.

In addition to its evolutionary implications, the trade-off between contact and transmissibility has epidemiological consequences as well. For example, is it possible for an intervention to paradoxically increase $\mathcal{R}_{0}$ ? Specific considerations include treatments that decrease symptom severity, such as antivirals for influenza [23], and vaccines that can affect the frequency of mild (e.g. asymptomatic) infection $[1,8,17,27,28,29,35]$. Whether this will be a practical concern for a specific disease of interest will depend upon the details of the disease and setting in question. For example, dose response relationships, relative pathogen shedding rates, and the spectrum of symptom severity will all impact the shape of $\mathcal{R}_{0}$, and how the reproduction number changes with intervention. These epidemiological features will affect inter- 
vention efficacy, and bear consideration when designing and implementing control efforts.

To illustrate, consider an intervention whose effect is solely to lessen symptom severity (increases $f$ ). Differentiating $\mathcal{R}_{0}$ with respect to $f$ gives

$$
\frac{\partial \mathcal{R}_{0}}{\partial f}=\mathcal{R}_{01}-\mathcal{R}_{00}
$$

Lessening symptom severity through intervention thus decreases the basic reproduction number when $\mathcal{R}_{00}>\mathcal{R}_{01}$, e.g. when severely symptomatic individuals dominate disease transmission. On the other hand, decreasing symptom severity will correspondingly increase contact rates. If this increase in contact rate outweighs the decrease in transmission probability, $\mathcal{R}_{0}$ will increase under intervention. This is particularly a concern for diseases with low infectious dose, and disease with high shedding rates for asymptomatic individuals.

\section{(a)}
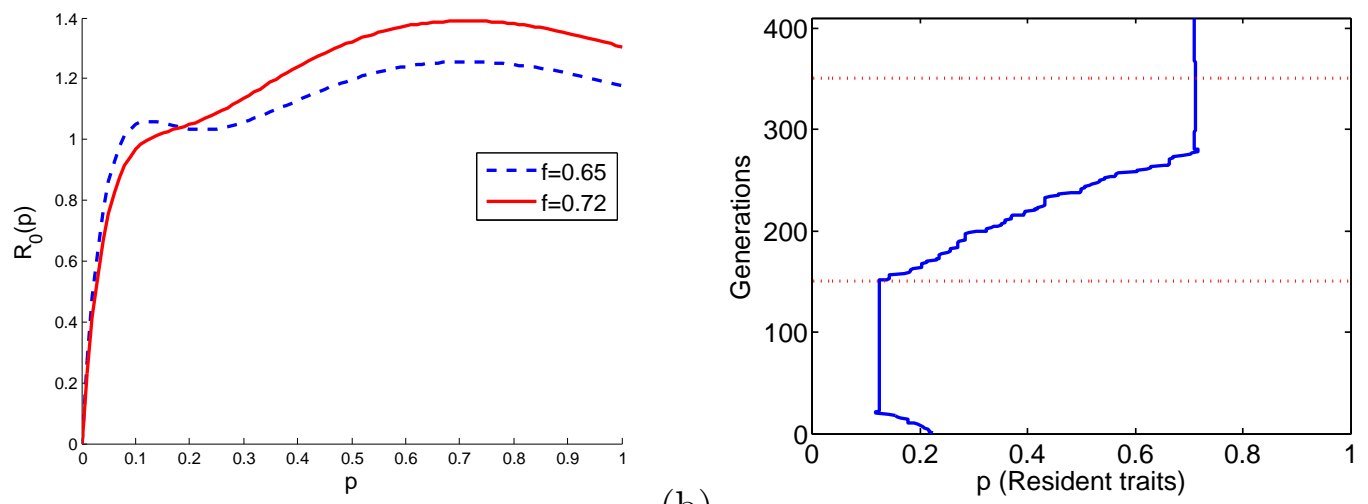

(b)

Figure 6: (a) A slight increase in the parameter $f$ leads to a dramatic change in the shape of $\mathcal{R}_{0}(p)$. When $f$ is increased from 0.65 to 0.72 , the number of critical points of $\mathcal{R}_{0}(p)$ changes from three to one. Contact function $c(p)=a \exp (-\lambda p)$, with parameter values $a=11.1, \lambda=14, d=0.0083, \gamma_{s}=\gamma_{m}=1 / 7, \sigma=0.1$. (b) Corresponding change in the evolutionary trajectory of $p$ due to change in $f$. The parameter $f$ is set at 0.65 for generation times before 150 and after 350 , and to 0.72 for generation times from 150 to 350. The trait $p$ first tends toward the local maximum (for $f=0.65) p=0.1234\left(\bar{p}_{a 1}\right.$ in the text) for generation times less than 150 , and subsequently converges to the global maximum (for $f=0.72$ ) $p=0.7111$ for generation times greater than 150 and less than 350. Next, the trait $p$ converges to the global maximum (for $f=0.65) p=0.7098\left(\bar{p}_{a 2}\right.$ in the text) for generation times greater than 350 . 
This may correspondingly lead to dramatically different evolutionary trajectories for $\mathcal{R}_{0}$ on longer time scales. To illustrate, consider the dotted curve in Figure $6 \mathrm{a}$, showing $\mathcal{R}_{0}$ in the absence of intervention. Here $f=0.65$, there are three critical points for the $\mathcal{R}_{0}$ curve, and the system lies near the local (but not global) maximum at $p=0.1234$ (Figure $6 \mathrm{~b}$ trajectory for generation times greater than 150). Now suppose that an intervention occurs at generation time 150 , changing $f$ to 0.72 . This changes the $\mathcal{R}_{0}$ curve to a unimodal shape with a single global maximum at $p=0.7111$, to which the system converges on an evolutionary time scale (Figure 6b). In fact this change persists even after intervention is lifted at generation 350, as the trait now converges to $\mathrm{p}=0.7098$ (the global maximum for the $f=0.65$ curve). Thus a small change in $f$ may lead to a large change in the trait value, due to a qualitative change of the shape of the $\mathcal{R}_{0}$ curve. In fact this example illustrates that a temporary intervention may result in a lasting increase in $\mathcal{R}_{0}$, due to the contact-transmissibility tradeoff.

One aspect this study highlights is the crucial need for empirical data on how illness affects contact patterns. The strength of the trade-off depends crucially upon how contact rates vary with symptom severity. Currently there is little empirical information describing this relationship, for example concerning the shape of how contact rates varies with symptom severity, or even measurement of relative contact rates for severely symptomatic to asymptomatic individuals. Recent efforts have recognized the importance of how disease status affects contact patterns [12], and empirical efforts are starting to take place aiming to measure these relationships [9, 34]. These data will be invaluable for understanding how trade-offs between transmissibility and contact affect disease dynamics on both epidemiological and evolutionary time scales.

\section{Acknowledgements}

This work was supported by the National Science Foundation through grant OCE-1115881 and the Mathematical Biosciences Institute (DMS-0931642). CJL acknowledges support from the Ministry of Science and Technology of Taiwan, R.O.C. (103-2917-I-564-003). The authors are grateful to the anony-

mous reviewers for their thoughtful, constructive comments, in particular for the suggestion to examine the effect of a transient intervention affecting $f$. 


\section{References}

[1] A. Aaby, P. Bhuiya, L. Nahar, K. Knudsen, A. de Francisco, and M. Strong. The survival benefit of measles immunization may not be explained entirely by the prevention of measles disease: a community study from rural Bangladesh. International Journal of Epidemiology, 32:106-116, 2003.

[2] R. M. Anderson and R. M. May. Coevolution of hosts and parasites. Parasitology, 85:411-426, 1982.

[3] A. Best and A. Hoyle. A limited host immune range facilitates the creation and maintenance of diversity in parasite virulence. Interface focus, 3(6):20130024, 2013.

[4] B. Boldin and É. Kisdi. On the evolutionary dynamics of pathogens with direct and environmental transmission. Evolution, 66(8):25142527, 2012.

[5] F. Brauer and C. Castillo-Chavez. Mathematical models in population biology and epidemiology, volume 1. Springer, 2001.

[6] H. J. Bremermann and H. R. Thieme. A competitive exclusion principle for pathogen virulence. Journal of Mathematical Biology, 27:179-190, 1989.

[7] J. J. Bull. Perspective - virulence. Evolution, 48:1423-1437, 1994.

[8] J. Castilla, P. Godoy, A. Domínguez, I. Martínez-Baz, J. Astray, V. Martín, M. Delgado-Rodríguez, M. Baricot, N. Soldevila, J. M. Mayoral, J. M. Quintana, J. C. Galán, A. Castro, F. González-Candelas, O. Garín, M. Saez, S. Tamames, and T. Pumarola. Influenza vaccine effectiveness in preventing outpatient, inpatient, and severe cases of laboratory-confirmed influenza. Clinical Infectious Diseases, 57(2):167$175,2013$.

[9] S.-C. Chen, S.-H. You, M.-P. Ling, C.-P. Chio, and C.-M. Liao. Use of seasonal influenza virus titer and respiratory symptom score to estimate effective human contact rates. Journal of Epidemiology, 22(4):353-363, 2012. 
[10] O. Diekmann. A beginner's guide to adaptive dynamics. Banach Center Publications, 63:47-86, 2004.

[11] O. Diekmann, J. A. P. Heesterbeek, and J. A. J. Metz. On the definition and the computation of the basic reproduction ratio $\mathcal{R}_{0}$ in models for infectious diseases in heterogeneous populations. Journal of Mathematical Biology, 28:365, 1990.

[12] E. P. Fenichel, C. Castillo-Chavez, M. G. Ceddia, G. Chowell, P. A. Gonzalez Parra, G. J. Hickling, G. Holloway, R. Horan, B. Morin, C. Perrings, M. Springborn, L. Velazquez, and C. Villalobos. Adaptive human behavior in epidemiological models. Proceedings of the National Academy of Sciences USA, 108(15):6306-6311, 2011.

[13] F. Fenner and F. Ratcliffe. Myxomatosis. Cambridge University Press, 1965.

[14] S. A. Frank. Models of parasite virulence. Quarterly Review of Biology, 71:37-78, 1996.

[15] V. V. Ganusov and R. Antia. Trade-offs and the evolution of virulence of microparasites: do details matter? Theoretical Population Biology, 64(2):211-220, 2003.

[16] S. A. H. Geritz, E. Kisdi, G. Meszena, and J. A. J. Metz. Evolutionarily singular strategies and the adaptive growth and branching of the evolutionary tree. Evolutionary Ecology, 12:35-57, 1998.

[17] H. Gulbudak and M. Martcheva. A structured avian influenza model with imperfect vaccination and vaccine induced asymptomatic infection. Bulletin of Mathematical Biology, 76(10):2389-2425, 2014.

[18] C. N. Haas, J. B. Rose, and C. P. Gerba. Quantitative microbial risk assessment. John Wiley, New York, 1999.

[19] J. B. Kaper, J. G. Morris, Jr., and M. M. Levine. Cholera. Clinical Microbiology Reviews, 8(1):48-86, 1995.

[20] B. R. Levin and J. J. Bull. Short-sighted evolution and the virulence of pathogenic microorganisms. Trends in microbiology, 2(3):76-81, 1994. 
[21] S. S. Long, C. J. Welkon, and J. L. Clark. Widespread silent transmission of pertussis in families - antibody correlates of infection and symptomatology. Journal of Infectious Diseases, 161(3):480-486, 1990.

[22] M. J. Mackinnon and A. F. Read. Genetic relationship between parasite virulence and transmission in the rodent malaria Plasmodium chabaudi. Evolution, 53:689-703, 1999.

[23] A. McGeer, K. A. Green, A. Plevneshi, A. Shigayeva, N. Siddiqi, J. Raboud, and D. E. Low. Antiviral therapy and outcomes of influenza requiring hospitalization in Ontario, Canada. Clinical Infectious Diseases, 45:1568-1575, 2007.

[24] J. Metz. Adaptive dynamics. In Encyclopedia of Theoretical Ecology. University of California Press: Berkeley, 2012.

[25] E. J. Nelson, J. B. Harris, J. Glenn Morris Jr., S. B. Calderwood, and A. Camilli. Cholera transmission: the host, pathogen and bacteriophage dynamic. Nature Reviews Microbiology, 7:693-702, 2009.

[26] K. Ozawa, T. Oka, N. Takeda, and G. S. Hansman. Norovirus infections in symptomatic and asymptomatic food handlers in Japan. Journal of Clinical Microbiology, 45(12):3996-4005, 2007.

[27] M. P. Preziosi and M. E. Halloran. Effects of pertussis vaccination on disease: vaccine efficacy in reducing clinical severity. Clinical Infectious Diseases, 37:772-779, 2003.

[28] B. J. Ridenhour, M. A. Campitelli, J. C. Kwong, L. C. Rosella, B. G. Armstrong, P. Mangtani, A. J. Calzavara, and D. K. Shay. Effectiveness of inactivated influenza vaccines in preventing influenza-associated deaths and hospitalizations among Ontario residents aged $\geq 65$ years: estimates with generalized linear models accounting for healthy vaccinee effects. PLOS ONE, 8:e76318, 2013.

[29] J. J. Schnorr, F. T. Cutts, J. G. Wheeler, S. M. Akramuzzaman, M. S. Alam, T. Azim, S. Schneider-Schaulies, and V. ter-Meulen. Immune modulation after measles vaccination of 6-9 months old Bangladeshi infants. Vaccine, 19:1503-1510, 2001. 
[30] J. L. Schulman. Experimental transmission of influenza virus infection in mice. IV. Relationship of transmissibility of different strains of virus and recovery of airborne virus in the environment of infector mice. Journal of Experimental Medicine, 125:479-488, 1967.

[31] E. Tronstein, C. Johnston, M.-L. Huang, S. Selke, A. Magaret, T. Warren, L. Corey, and A. Wald. Genital shedding of herpes simplex virus among symptomatic and asymptomatic persons with HSV-2 infection. Journal of the American Medical Association, 305(14):1441-1449, 2011.

[32] M. van Baalen. Coevolution of recovery ability and virulence. Proceedings of the Royal Society of London B: Biological Sciences, 265(1393):317-325, 1998.

[33] P. van den Driessche and J. Watmough. Reproduction numbers and sub-threshold endemic equilibria for compartmental models of disease transmission. Mathematical Biosciences, 180:29-48, 2002.

[34] K. van Kerckhove, N. Hens, W. J. Edmunds, and K. T. D. Eames. The impact of illness on social networks: implications for transmission and control of influenza. American Journal of Epidemiology, 178(11):1655$1662,2013$.

[35] A. Vila-Corcoles, O. Ochoa-Gondar, C. Llor, I. Hospital, T. Rodriguez, and A. Gomez. Protective effect of pneumococcal vaccine against death by pneumonia in elderly subjects. European Respiratory Journal, 26:1086-1091, 2005.

[36] A. L. Vivas-Barber, C. Castillo-Chavez, and E. Barany. Dynamics of an "SAIQR" influenza model. BIOMATH, 3(2):1409251, 2014.

[37] D. Waxman and S. Gavrilets. 20 questions on adaptive dynamics. Journal of evolutionary biology, 18(5):1139-1154, 2005. 